\title{
Editorial
}

\section{Engineering Nanostructures of Inorganic Materials for Optical and Chemical Applications}

\author{
Yong Yang, ${ }^{1}$ Masayuki Nogami, ${ }^{1}$ Zhengren Huang, ${ }^{1}$ and Yongsheng $\mathbf{L i}^{2}$ \\ ${ }^{1}$ State Key Laboratory of High Performance Ceramics and Superfine Microstructure, Shanghai Institute of Ceramics, \\ Chinese Academy of Sciences, Shanghai 200050, China \\ ${ }^{2}$ East China University of Science and Technology, Shanghai 200237, China
}

Correspondence should be addressed to Yong Yang; yangyong@mail.sic.ac.cn

Received 26 November 2013; Accepted 26 November 2013

Copyright (C) 2013 Yong Yang et al. This is an open access article distributed under the Creative Commons Attribution License, which permits unrestricted use, distribution, and reproduction in any medium, provided the original work is properly cited.

Considerable interest is being exhibited in the novel and enhanced properties of nanostructured materials. These materials, with their constituent phase or grain structures modulated on a length scale of less than $100 \mathrm{~nm}$, are now artificially synthesized by a wide variety of physical, chemical, and mechanical methods. Today, scientists and engineers have access to a wealth of technologies such as self-assembly, chemical template, electron-beam lithography, focusedion-beam (FIB) lithography, nanoimprint, nanomolding, and scanning-probe lithography to build nanostructures at unprecedented scales, resolution, and throughput. The art of developing functionalized nanostructured materials exploiting unusual interfacial properties seems to have produced hitherto unknown man-made materials. Exploitations of nanostructured metal and oxide materials with many excited optical, chemical, and mechanical properties will lead to the broad range of potential applications including catalyst, drug discovery, sensing, nanoimaging, spectroscopy, optoelectronics, data storage, and structured-materials with enhanced mechanical properties.

In this special issue, a series of contributed papers is focused on the function-motivated nanostructure design, fabrication, characterization, and application including metal, oxide, and ceramics. A wide range of recent progress on the chemical and physical fabrication methods of metal nanostructures, their optical properties, and application has been reviewed by G. Kawamura's and Y. Yang's groups. C. T. Torres et al. reported the transmittance modulation of optical signals in a nanocomposite integrated by two different silver doped zinc oxide thin solid films. T. K. Lee et al. reported polyol-free synthesis of highly loaded $\mathrm{Pt}$ catalysts on sulfuric-acid-treated graphene oxide (SGO) and their catalytic properties. Y. H. Lin's group reported Fe-doped $\mathrm{NiO}$ nanofibers synthesized by electrospinning method and studied their ferromagnetic behaviors. The surface modification of $\mathrm{SiC}$ ceramics and the optical application have been reviewed by $\mathrm{F}$. Jiang. The nanostructures of $\mathrm{Si}, \mathrm{GaN}$, and $\mathrm{TiO}_{2}$ and their optical-electric properties were studied by $\mathrm{D}$. Li et al., S. P. Chang et al., and L. Cheng et al., respectively. Kasuga's group demonstrated aluminum silicate nanotube coating of siloxane-poly (lactic acid)-vaterite composite fibermats by electrospinning. This approach may provide a new method of improving the surface of polymer-based biomaterials.

\section{Acknowledgments}

We would like to express our sincere appreciation to all the authors and coauthors and to all the reviewers for their contribution to this special issue. We also thank the reviewers for their time and valuable comments.

Yong Yang Masayuki Nogami Zhengren Huang Yongsheng Li 

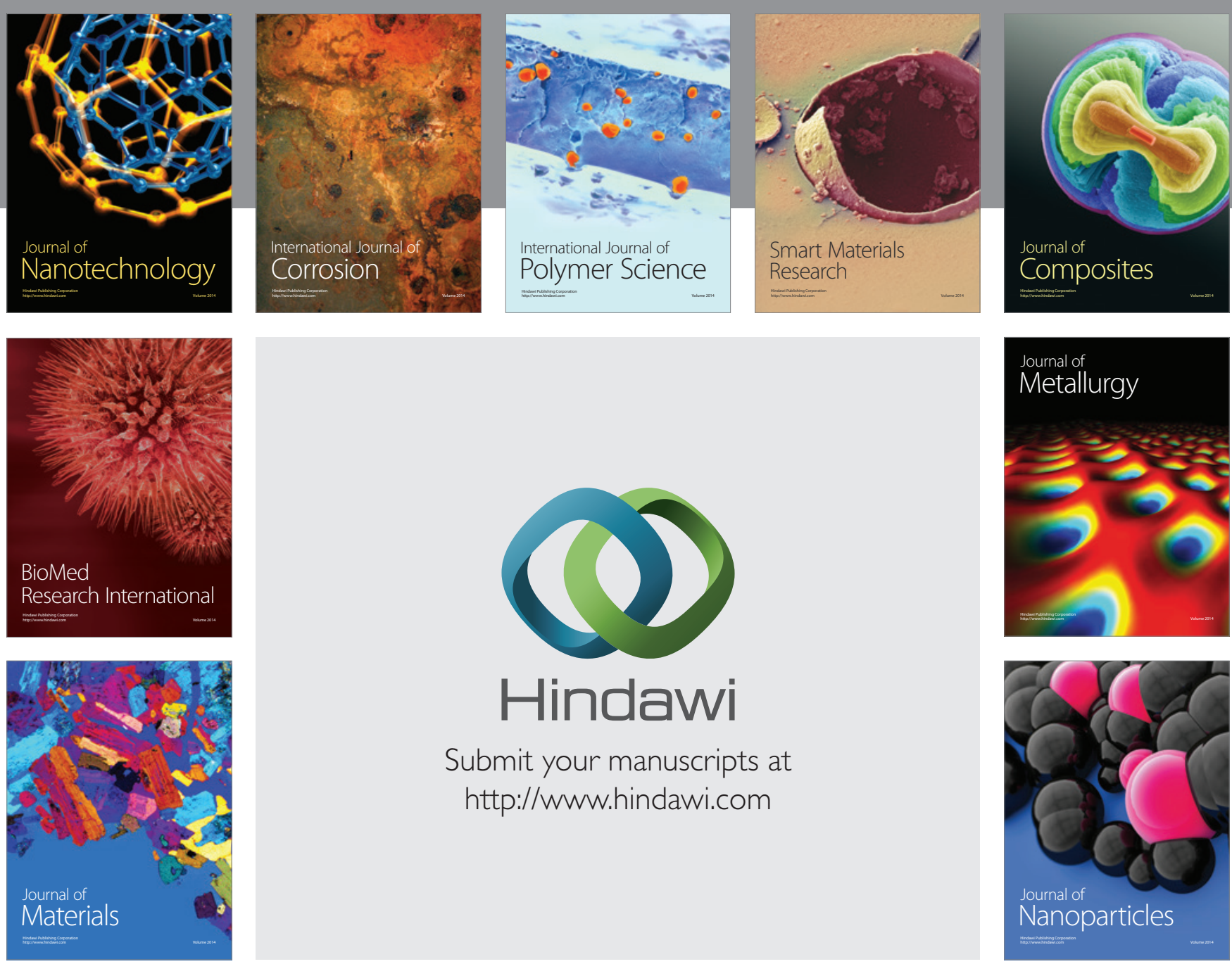

Submit your manuscripts at http://www.hindawi.com
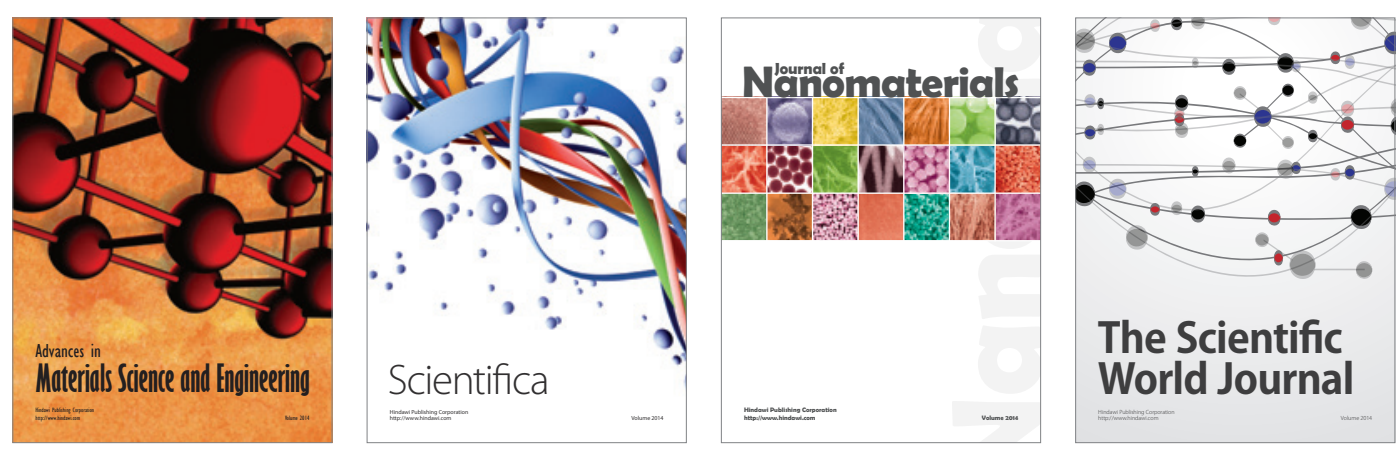

\section{The Scientific World Journal}
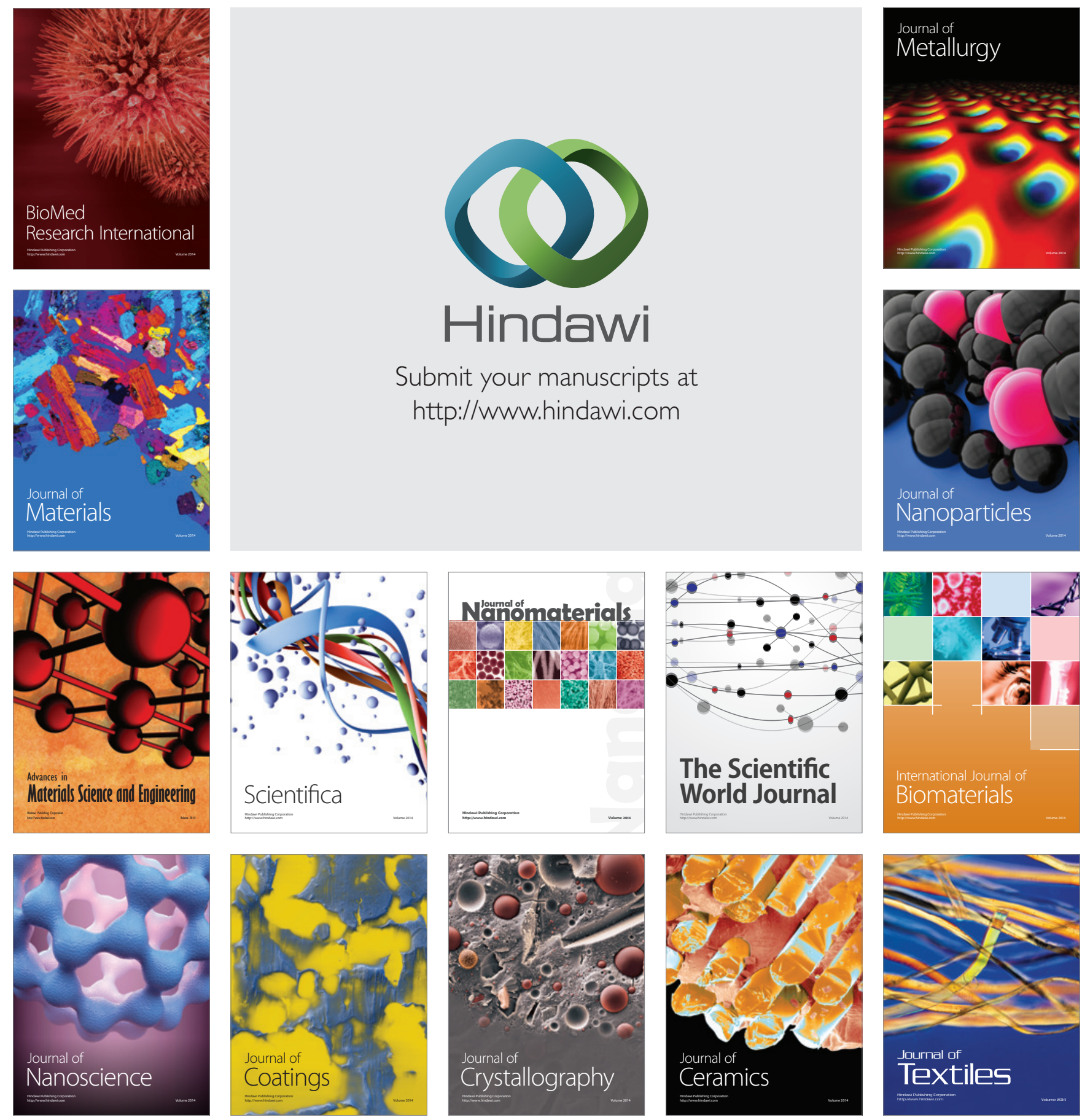